\title{
Activity and Kinematics of Late M, L, and T Dwarfs
}

\author{
James Liebert \\ Department of Astronomy, The University of Arizona, Tucson, AZ, \\ USA 85721
}

\begin{abstract}
The kinematics of late $\mathrm{M}$ and certainly $\mathrm{L}$ dwarfs indicate that they have a mean age of no more than a few Gyr. This result does not rule out a mix of young brown dwarfs and ZAMS stars. Relative $\mathrm{H} \alpha$ emission strengths begin to decline in the late $M$ dwarfs, even though about $100 \%$ of the M7-8 dwarfs show the weaker emission. Both the frequency and likely strength of this emission declines with spectral type among the L's and T's. A larger sample of late $M$ and $L$ dwarfs, which may provide more definitive tests of the kinematic and activity properties, is being assembled from our NStars project.

Two unusual objects, one an M9.5e and the other a T6e, show persistent $\mathrm{H} \alpha$ emission at least an order of magnitude stronger than their counterparts of similar spectral type. If these are hypothesized to be very young stellar objects ( $\lesssim 10 \mathrm{Myr}$ ) like some observed in Rho Oph, Sigma Ori and Taurus - evolutionary models would require them to have very low masses: In particular, for an entity to fade to a late $\mathrm{T}$ dwarf temperature and luminosity within $10 \mathrm{Myr}$, its mass should be no more than several Jupiters. This seems implausible for an isolated object not near any obvious site of recent star formation. However, our attempt to show that the emission might be due to accretion in a binary system was unsuccessful.
\end{abstract}

\section{Introduction}

It is well known that the evolutionary tracks of young, substellar brown dwarfs nearly coincide with the essentially static positions of low mass hydrogen burning stars (the end of the ZAMS). In fact it is not yet clear where the ZAMS terminus actually lies in, for instance, spectral type. This must be a matter of definition. It has been clear since D'Antona and Mazzitelli (1985), that stellar entities near this "edge" may undergo substantial hydrogen-burning for up to several Gyrs, though not quite managing $100 \%$ of the required pressure support to stop completely the contraction. Such "transition mass" objects (TMOs) as D'Antona and Mazzitelli first called them, may undergo nuclear burning for longer than do main sequence F stars. Eventually, however, the TMOs succumb to electron degeneracy and conductive energy transport. Once this more efficient transport causes the central temperature to edge downward, the nuclear reactions are quickly quenched, and the object winds up as a brown dwarf. 
It has therefore been clear that late $\mathrm{M}$ dwarfs should be a mixture of stellar and a limited fraction of substellar entities. The ZAMS edge appears to be somewhere at some mid-L type, for solar composition. The likely multi-Gyr age of GD 165B (Kirkpatrick et al. 1999) indicates that L4 dwarfs may include either TMOs or ZAMS stars. Thus, early L dwarfs are a different mix. Somewhere in the late $\mathrm{L}$ range, the ubiquity of lithium $(6707 \AA)$ detections indicates that all are substellar, as must be the $\mathrm{T}$ dwarfs.

For so-called field objects - unattached to systems of known age - the clues to their masses and stellar/substellar nature include kinematics, rotation, possible dynamo activity, as well as of course the lithium feature. The only feasible measure of chromospheric activity is the $\mathrm{H} \alpha$ emission line (or its absense). However, the presense of strong $\mathrm{H} \alpha$ emission might be caused by something other than a dynamo. In the next section, I summarize briefly two recent studies of the kinematics. Following that, I review the incidence and strength of $\mathrm{H} \alpha$ in our 2MASS sample of M, L and T dwarfs. The discovery of a few objects with sustained emission of unusual strength pose an unsolved mystery. Finally, I mention the role our current NStars project in discovering more solar neighbors of very low mass, missed in proper motion surveys, but amenable to the detailed studies the nearest and brightest of a given class can best offer.

\section{Kinematics}

Even with a 10 meter aperture telescope, only the nearest late $M$ and (barely) early L dwarfs may be studied at high spectral resolution at optical wavelengths. However, Reid et al. (2002) were able to measure radial velocities and hence obtain the complete space motions of a sample of 39 M6.5-L0.5 2MASS stars - the (Keck) HIRES sample. The velocity dispersions of the U,V,W Galactic components are similar to the large sample of catalogued dMe dwarfs spanning all M subtypes studied by Hawley, Gizis and Reid (1996), but are significantly smaller than their larger sample of $\mathrm{dM}$ stars which lack $\mathrm{H} \alpha$ emission. This suggests that these very late dwarfs have a mean age of 1-2 Gyr, as was inferred for the dMe stars.

For the dMe dwarfs, the interpretation is that the line emission fades after 1-2 Gyr. Late $\mathrm{M}$ dwarfs have a nearly $100 \%$ incidence of $\mathrm{H} \alpha$ appearing in emission (even at the moderate spectral resolution we have typically used to classify the 2MASS sources) - see Gizis et al.(2000). The interpretation for these is more complicated. Late $M$ dwarfs are known to include objects with old disk kinematics, which should characteristically have ages of several Gyr or more. The velocity dispersions suggest an admixture with a population of substellar, and necessarily young, objects. However, our detection of lithium in only two of the HIRES sample puts a limit on this fraction. Surely the sample also has a kinematic bias - it excludes catalogued proper motion stars of presumably higher velocities, because the sample consists of new 2MASS discoveries. The sample is regrettably too small for firm conclusions. Infrared high resolution spectra can be used to assemble a substantially larger sample of such sources with complete kinematics in the future.

More limited kinematics from tangential velocities only are available for a sample of mostly L dwarfs with new trigonometric parallaxes discussed by Dahn 


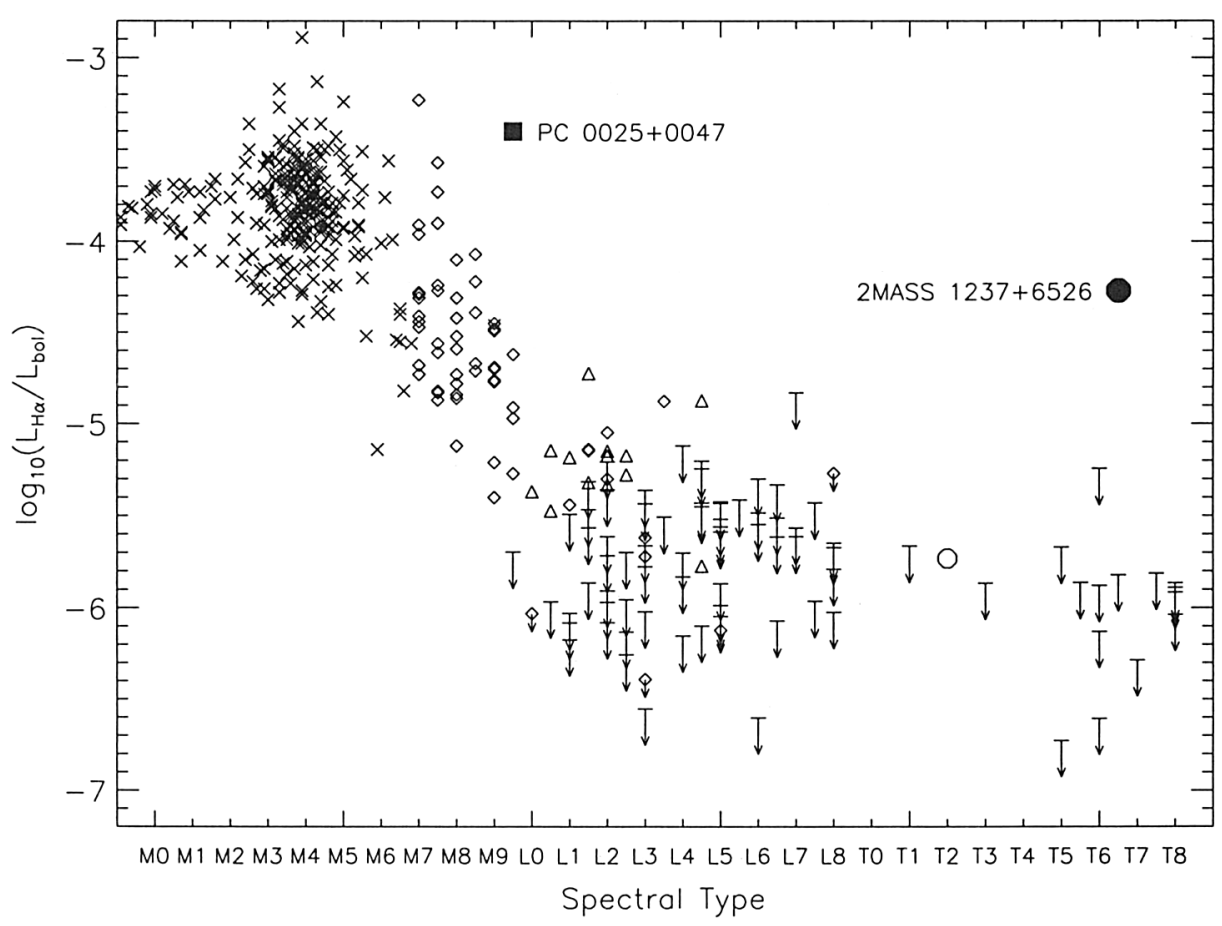

Figure 1. Ratio of $\mathrm{H} \alpha$ luminosity to bolometric versus spectral type for cool dwarfs. Also shown are M and L dwarf data from Hawley, Gizis and Reid (1996, crosses) and Gizis et al. (2000, diamonds). T dwarfs are from Burgasser et al. (2002a).

et al. (2002). The parallaxes of course ensure that the proper motions may be accurately converted into tangential velocities. The mean values for these are similar to the Hawley, Gizis and Reid dMe sample, and smaller than the dM sample, pretty much as expected.

\section{3. $\mathbf{H} \alpha$ Emission}

$\mathrm{H} \alpha$ emission is usually a measure of chromospheric activity for a solar or latetype star. Chromospheric activity is one form of energy release by the magnetic dynamo which spins down the rotation of the star. However, the classic Parker theory for the Sun relies on the presence of a radiative core to which the magnetic field is anchored. At middle- to late-M masses, the star becomes completely convective, and there is much in the literature about how the dynamo mechanism is expected to change or weaken. It is not clear whether dynamo breaking is effective at all for the $\mathrm{L}$ and $\mathrm{T}$ spectral types.

In Figure 1, adapted from Gizis et al. (2000), the surprisingly behavior of $\mathrm{H} \alpha$ fluxes relative to the bolometric fluxes are plotted against the spectral 
types - presumably a proxy for the stellar $\mathrm{T}_{\text {eff }}$. The ratio is a reasonable measure of the dynamo's strength - its energy release compared to the interior luminosity. It has long been known for that, for the most active stars from one solar mass downward, this logarithmic ratio is between -4 and -3 . Cooler than M5, for the large majority at least, this ratio begins to fall. Ironically, the fraction of the $\mathrm{M}$ dwarfs showing $\mathrm{H} \alpha$ emission begins to increase rapidly, and reaches essentially $100 \%$ for M7 and M8 spectral types, even though their level of activity as indicated by this ratio is nearly an order of magnitude less than the most active middle-M dwarfs.

For the M9 and the L dwarfs both the fraction showing emission and the level of activity plummet. Only upper limits are available for late $\mathrm{L}$ dwarfs near the activity level of the few early $\mathrm{L}$ detections. The majority of $\mathrm{T}$ dwarfs seem to behave similarly as the later L's. Mohanty et al. (2002) offer a plausible explanation for the ineffectiveness of the magnetic dynamo when the outer envelopes become neutral, and this may indeed happen for $\mathrm{L}$ and $\mathrm{T}$ dwarfs.

\section{Two Spectacular Exceptions}

The two large filled symbols exhibiting enhanced levels of activity relative to their peers are PC 0025+0047(=PC0025), classified M9.5e (Schneider et al. 1991), and the T6e dwarf 2MASS 1237+6526(=2M1237, Burgasser et al. 2000). These show $\mathrm{H} \alpha$ emission fluxes an order of magnitude or more stronger than those counterparts with detected emission. Occasionally, $\mathrm{H} \alpha$ fluxes of similar strength have been seen in brief flares. 2MASS $0149090+295613$, with the same spectral type as the PC object, showed $\mathrm{H} \alpha$ of similar strength in a single 600second exposure, after which the flare faded (Liebert et al. 1999). Another such object - a middle L dwarf - is presented by D. Kirkpatrick (this conference). In contrast, the PC0025 and 2M1237 objects have exhibited unusually strong $\mathrm{H} \alpha$ at every observation.

Martín, Basri and Zapatero Osorio (1999) have discussed repeated observations of $\mathrm{PC} 0025$ in which the continual $\mathrm{H} \alpha$ emission is documented. Noting the observed weakness of its atomic resonance lines, these authors favored the interpretation that the object is young and well above the ZAMS. The fragile trigonometric parallax published in Dahn et al. (2002) suggests that the entity is a bit overluminous relative to other M9-L0 dwarfs. Indeed $\mathrm{H} \alpha$ emission this persistent and strong is observed in some of the lowest mass objects in very young ( $\$ 10 \mathrm{Myr}$ ) clusters and associations such as Rho Ophiuchus, Sigma Orionis and the Taurus clouds. I am not aware of very low mass objects in older clusters such as Alpha Persei or the Pleiades exhibiting such strong emission. Were PC0025 as young as $10 \mathrm{Myr}$, however, the calculations of Burrows et al (2002, see Fig. 8) suggest that it should be barely massive enough to fuse deuterium $\left(15-20 \mathrm{M}_{J}\right)$. For $2 \mathrm{M} 1237$ to have faded to a likely $\mathrm{T}_{\text {eff }}$ below $1,000 \mathrm{~K}$ within $10 \mathrm{Myr}$, the models suggest it has a mass of $3-4 M_{J}$ ! The existence of such an isolated object may be possible as an ejected planetary embryo (Reipurth and Clarke 2001, and Reipurth, this conference), however its existence far from any apparent site of very recent star formation renders such an age for it implausible.

Such reservations led Burgasser et al. (2002b) to pursue other possible origins for the line emission, with regard to $2 \mathrm{M} 1237$. We searched for variability, 
in particular for evidence that the entity is some kind of interacting binary perhaps a hydrogen degenerate counterpart to the AM CVn accreting binary white dwarfs. However, we found absolutely no evidence in favor. Rather, it appears unlikely that the 2MASS object is binary.

In conclusion, no explanation for the enhanced $\mathrm{H} \alpha$ fluxes of these objects is apparent. They may somehow be significantly older than the $\mathrm{H} \alpha$ stars in the very young clusters. It is also possible that the evolutionary models predict incorrect luminosities and temperatures at ages as young as $10 \mathrm{Myr}$.

\section{NStars}

I conclude with a few remarks about our relatively-new "NStars" project, and its potential to resolve these questions with larger samples. Kelle Cruz (poster paper, this conference) describes this project, led by herself and Neill Reid (Reid and Cruz 2002). Briefly, we are matching 2MASS infrared point sources with those from the POSS and other optical Schmidt surveys. In less than two years, the result has been the discovery of hundreds of new late $M$ and early $L$ dwarfs, newly-discovered solar neighbors. They are previously uncataloged because the proper motions are below the catalog limits of Luyten surveys. Some are within the 20-25 parsec distances that traditionally place them in the Catalog of Nearby Stars maintained by $\mathrm{H}$. Jahrreiss (Astronomische Rechenzentrum, Heidelberg). One is a possible strong dMe with sustained $\mathrm{H} \alpha$ emission, perhaps another rare example of the phenomenon discussed in the previous section.

Acknowledgments. The results discussed here of course include leading contributions from my principal collaborators on the 2MASS and NStars teams - Adam Burgasser, Kelle Cruz, Conard Dahn, John Gizis, Hugh Harris, Davy Kirkpatrick, and Neill Reid. I also appreciate useful discussions with Adam Burrows and Michael Meyer. This research is supported by a NASA JPL grant (961040NSF).

\section{References}

Burgasser, A.J., Kirkpatrick, J.D., Brown, M.E., Reid, I.N., Burrows, A., Liebert, J., Matthews, K., Gizis, J.E., Dahn, C.C., Monet, D.G., Cutri, R.M., and Skrutskie, M.F. 2002a, ApJ, 564, 421

Burgasser, A., Kirkpatrick, J.D., Reid, I.N., Liebert, J., Gizis, J.E., and Brown, M.E. 2000, AJ, 120, 473

Burgasser, A., Liebert, J., Kirkpatrick, J.D., and Gizis, J.E. 2002b, AJ, 123, 2744

Burrows, A., Hubbard, W.B., Lunine, J.I. and Liebert, J., 2001 Rev. Mod. Phys., v. 73, p. 719

Dahn, C.C., Harris, H.C., Vrba, F.J., Guetter, H.H., Canzian, B., Henden, A.A., Levine, S.E., Luginbuhl, C.B., Monet, A.K.B., Monet, D.G., Pier, J.R., Stone, R.C., Walker, R.L., Burgasser, A.J., Gizis, J.E., Kirkpatrick, J.E., Liebert, J., and Reid, I.N. 2002, AJ, in press

D'Antona, F., and Mazzitelli, I. 1985, ApJ, 296, 502 
Gizis, J.E., Monet, D.G., Reid, I.N., Kirkpatrick, J.D., Liebert, J., and Williams, R.J. 2000, AJ, 120, 1085

Hawley, S.L., Gizis, J.E., and Reid, I.N. 1996, AJ, 112, 2799

Kirkpatrick, J.D., Allard, F., Bida, T., Zuckerman, B., Becklin, E.E., Chabrier, G. and Baraffe, I. 1999, ApJ, 519, 834

Liebert, J., Kirkpatrick, J.D., Reid, I.N., and Fisher, M.D. 1999, ApJ, 519, 345 Martín, E.L., Basri, G., and Zapatero Osorio, M.R. 1999, AJ, 118, 1005

Mohanty, S., Basri, G., Shu, F., Allard, F., and Chabrier, G. 2002, ApJ, 571, 469

Reid, I.N., Kirkpatrick, J.D., Liebert, J., Gizis, J.E., Dahn, C.C., and Monet, D.G. 2002, AJ, 124519

Reid, I.N., and Cruz, K.L. 2002, AJ, 123, 2806

Reipurth, B., and Clarke, C. 2002, AJ, 122, 432

Schneider, D.E., Greenstein, J.L., Schmidt, M., and Gunn, J.E. 1991, AJ, 102, 1180 